\title{
VEGETATIVE PROPAGATION OF Scyphiphora hydrophyllacea GAERTN F. FOR CONSERVATION
}

\author{
P. L. Hettiarachchi ${ }^{1}$, P. A. G. W. Premathilake 1 \& S. Hettiarachi ${ }^{2}$ \\ 1Department of Botany, University of Sri Jayewardenepura \\ 2 Department of Botany, University of Ruhuna
}

Scyphiphora hydrophyllacea is a highly threatened true mangrove confined to Jaffna and a single locality in private land in Kalpitiya, Sri Lanka. It produces fruits and seeds, but seedlings and young plants are absent in the natural habitat. Conservation has become cssential and urgent to prevent local extinction of the species. Seed germination using moist filter paper method with habitat water varying from $0 \%-100 \%$ concentration totally failed in a previous study. This investigation therefore, aims at the possibility of propagating $S$. hydrophyllacea by vegetative means viz. stem cuttings, girdle cuttings and air layering. Stem cuttings of three stages of maturity (first 5 nodes, 6-9 nodes, 10-13 nodes) were used. Distilled water, water from natural habitat and its $75 \%, 50 \%$ to and $25 \%$ dilutions with and without IAA and activated charcoal $(0.25 \mathrm{~g} / 100 \mathrm{ml})$ were tested for rooting. Further, dipping of the cut base of the cuttings in commercial rooting powders namely Secto containing NAA, Seridox and Clonex both containing IBA and different concentrations of IAA $(0.5 \mathrm{mg} / 1-2.0 \mathrm{mg} / 1)$ prior to leaving for rooting was also carried out. Air layering was done by enclosing one-month-old girdles in moist rooting media (three different mixtures and habitat soil). After one month $50 \mathrm{ml}$ of half strength Hogland solution was injected to the medium. Another set of layers were treated by applying a paste of Secto on the trimmed edge of the girdle, mixing the same powder with the rooting medium and injecting IAA to the root bail after one month. Rooted cuttings, girdles and layers were transferred to soil substrate in pots and maintained under green house conditions. Rooting was observed only in distilled water in both stem and girdle cuttings. There was no significant effect by exogenous IAA and commercial rooting powders. Charcoal improved rooting from girdle cuttings up to $75 \%$. Stage 11 (6-9 nodes from the apex) cuttings gave highest percentage $(40 \%)$ of rooting. A maximum of $60 \%$ of layers had rooted in one month. Use of IAA and rooting powder did not improve the rooting percentage in layers. The plantlets obtained are established in pots in the green house under natural temperature and light. The results satisfactorily show that $S$. hydrophyllacea can be vegetatively propagated by using appropriate methods. This is important in conservation, as they do not produce seedlings from seeds. Experiments in hardening of the plantlets to grow in their natural habitat are in progress.

Proceedings of the Eighth Annual Forestry and Environment Symposium 2002 of the Department of Foresiry and Environmental Science, University of Sri Jayewardenepura, Sri Lanka 